\title{
Visual detection as a function of attentional demand and perceptual system error
}

\author{
STUART M. KEELEY ${ }^{2}$ \\ BOWLING GREEN STATE UNIVERSITY
}

\begin{abstract}
Attentional demands were varied in a two-alternative, forced-choice detection experiment. $A$ bar indicator designated the target form in one condition and occurred in random locations in a second condition. Exposure durations necessary for predetermined single-form display HRs were determined for each of eight $\mathrm{Ss}$ to measure performance at different levels of perceptual system error. Forms differed in only a single feature. Detection was superior when the bar indicator designated the target form, and differences increased with increased display size. Evidence for interference in detection due to the presence of nontarget forms apart from noting requirements of such forms was found. Estimates of number of perceptual channels noted did not clearly differentiate serial from parallel processing models. It was concluded that display interference error and spatial selectivity influences are important determinants of detection accuracy.
\end{abstract}

There has been an increasing trend toward considering information processing from tachistoscopic displays in terms of several subprocesses (e.g., Sperling, 1963; Neisser, 1967). The se include the arrival of visual information over sensory channels where it persists centrally as a stimulus trace, or as an icon (Neisser, 1967), a noting response that scans or analyzes the available input, and the encoding of certain information into short-term memory.

Recent experiments have used forced-choice detection techniques to investigate the noting subprocess (e.g., Estes \& Taylor, 1964, 1966; Estes \& Wessell, 1966). These investigations have focused on the rate of processing and the question of whether the noting process operates in serial or parallel. A primary advantage to their methodology is that it avoids memory store limitations. However, since the detection task does not require the storage of nontarget forms, the term noting is used here only to refer to a decision-making operation on input items. This operation may or may not lead to the encoding or storage of the items.

Detection methodology has been insensitive to certain possible processing influences attributable to characteristics of the stimulus forms, to temporal factors, and to non-noting subprocesses, which have made it difficult to interpret the evidence in terms of serial or parallel processing.

One problem with previous methodology has been the use of letters as stimulus forms. Interpreting detection methodology estimates of the number of forms noted as evidence for either serial or parallel noting requires the assumption that nontarget forms are noted. The use of multidimensional forms (letters) creates difficulties, since evidence is accumulating that some processing occurs at the level of features rather than forms (Neisser, 1967; Keeley \& Doherty, 1968). With letters, the visual detection task may be requiring differing degrees of analysis for target and nontarget forms, and for forms differing in number of discriminative features. If such hierarchical levels of analysis occur, detection tasks using multifeatured forms would appear to be insensitive to differences between serial and parallel models for the noting of forms. Therefore, in the present investigation target and nontarget forms for a forced-choice detection task were selected that differ among themselves on the basis of a single feature.

A second difficulty with previous detection studies is the failure to consider the duration and/or luminance required to process a single form. By controlling the detection accuracy for any single form falling at a given foveal location so that the hit rate (HR) is greater than chance but less than $100 \%$, a sensitive measure of the energy level required to note a single form can be obtained (Eriksen, 1966; Collins \& Eriksen, 1968). When exposure duration and luminance are set at a value where $S$ is $100 \%$ correct when a single form is presented, it is impossible to determine to what degree energy could be reduced and $\mathrm{S}$ still maintain a $100 \%$ criterion.

The use of a less than $100 \%$ single form $\mathrm{HR}$ also permits the computation of the probability of a given form being available through sensory channels for noting. If perceptual errors are uncorrelated for forms presented to different foveal locations and an assumption is made that the errors involved in detecting a single form are associated with sensory channels and not the noting process, the HR corrected for chance can be viewed as an estimate of the probability of a form being available to the noting mechanism on any given trial. Evidence indicates that separation of $1 \mathrm{deg}$ or more on the retina eliminates correlated perceptual error (Collins \& Eriksen, 1968).

Even if the above two changes in methodology are incorporated in the detection task, to determine adequately the extent to which changes in detection accuracy are affected by noting requirements per se, one must give consideration to the effect of non-noting requirements on detection of the target form. Evidence suggests that a loss in identifiability of single forms may occur due to a disturbance in attentional, or preattentional, mechanisms caused by the presence of additional forms in the display (Eriksen \& Lappin, 1967). If such a loss occurs as a result of non-noting factors, knowledge of such a reduction in accuracy would appear critical for determining whether noting is serial or parallel.

One means of assessing whether changes in HR are due to noting requirements or to non-noting influences is to observe changes that occur when experimental conditions demand different noting operations on the nontarget forms. This is accomplished in the present investigation by varying attentional demands while holding the available information and response uncertainty constant. Differences in detection accuracy in the two conditions provide an estimate of limitations due to noting requirements. If processing is serial, rate of noting of nontarget forms should be the primary limitation to processing. If processing is parallel, the number of independent sensory channels should be the primary limitation, and noting requirements for nontarget forms should not affect performance except when the number of forms exceeds the number of parallel channels.

Apparatus and Stimuli

\section{METHOD}

A three-field tachistoscope was used for stimulus presentations. 
All three fields were backlighted. Preexposure and postexposure fields were dark. A faintly glowing fixation cross was presented in one field. Stimuli were presented in the second field, and indicator pointers (as further explained below) were presented in the third field. The stimuli and indicator were presented simultaneously for equal durations at a luminance of 1.4 apparent $\mathrm{ft}$-c.

To assure that discrimination of all forms, target and noise, was dependent on a single characteristic, Landolt Cs were selected as the stimulus forms. Each $\mathrm{C}$ varied only in the direction of its gap. The gap could occur in any one of four directions: up, down, left, or right.

The stimuli were circular displays of $1,3,4,5,6$, or 8 forms. These displays were photographic negatives and when transilluminated give light figures with $99 \%$ contrast. The form locations were the 22.5-, 67.5-, 112.5-, 157.5-, 202.5-, 247.5-, 292.5-, and $337.5-\mathrm{deg}$ positions on an imaginary circle (considering the top to be $0 \mathrm{deg}$ ). The radius from a central fixation point was set at such a distance as to allow eight $\mathrm{Cs}$ to be placed in the array simultaneously and be separated by 1 deg of visual angle. Viewed through the tachistoscope, the $\mathrm{Cs}$ were at a radius of $2 \mathrm{deg}$ of visual angle from the central fixation point. Each $\mathrm{C}$ subtended $.3 \mathrm{deg}$ of visual angle. The gap subtended $.06 \mathrm{deg}$.

Each display contained one of two possible target forms plus the appropriate number of noise forms. The target forms were left-gap and right-gap Cs. The noise forms were up-gap and down-gap Cs.

One set of eight stimulus cards was constructed for single-form (SF) displays, providing stimuli with a right- and left-gap target form in each position, since the cards could be turned over to produce the reverse gap positions. Two sets of eight cards each were made up for the 3-, 4, 5-, 6-, and 8-form displays. Locations for the extraneous forms were randomly assigned with the constraint that in 3-form displays, all forms occurred in different quadrants; and in all displays of greater than 3 forms, at least one form fell into each of the four quadrants of the circle.

For every stimulus presentation, a pointer appeared just outside the display, indicating one of the eight locations. The pointers were narrow white lines, $50 \mathrm{~min}$ of visual angle in length. The pointer extended to within $1 / 2 \mathrm{deg}$ of visual angle of the designated form. Eight indicator location cards were constructed, one for each of the possible display locations.

\section{Procedure}

Eight students, enrolled at Danville (Illinois) Junior College, participated in this experiment as paid volunteers. Viewing was monocular, and all Ss had normal vision in the eye utilized during the experiment.

Each $S$ had three practice sessions. During these sessions exposure durations were determined for each $S$ that would yield 75 and $90 \%$ HRs for SF displays. The mean exposure durations for the eight Ss were 3.75 and $6.50 \mathrm{msec}$ for the 75 and $90 \% \mathrm{SF}$ HR conditions, respectively.

Experimental sessions consisted of presenting randomly 1-, 3-, 4-, 5-, 6-, and 8-form displays at the appropriate SF exposure duration. During the session, $5 \mathrm{~min}$ of dark adaptation was followed by 10 practice judgments and 96 test judgments. For each trial, $S$ was instructed to fixate on the faintly glowing cross until clearly focused, trigger the exposure, and report whether the gap of the target form was left or right.

Attentional demand varied according to the relevance of a bar

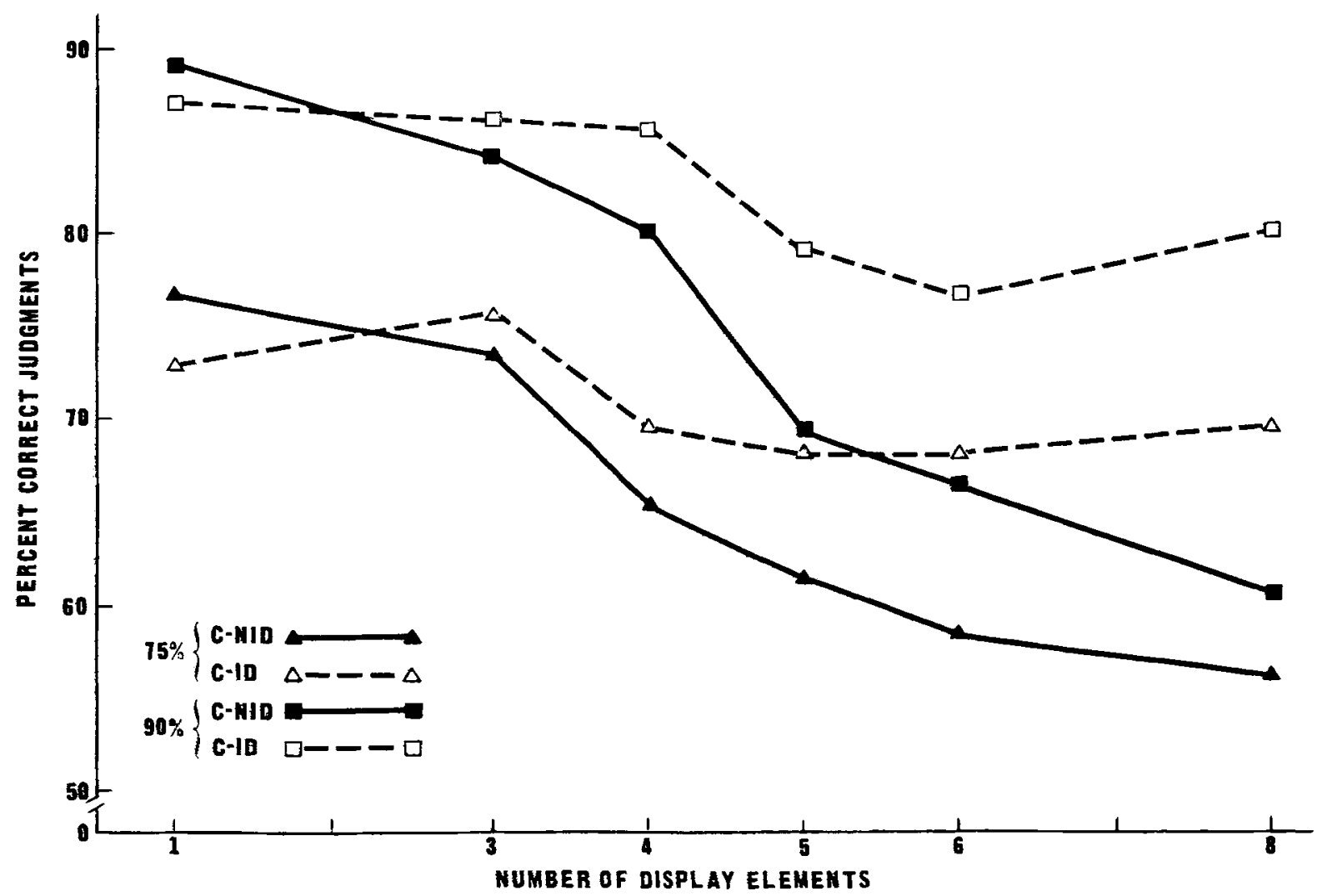

Fig. 1. Per cent correct judgments obtained at the $75 \%$ and $90 \%$ single-form HR exposure durations under indicator relevant (ID) and indicator irrelevant (NID) conditions as a function of the number of display forms. 
indicator. In one condition (ID) the target form was always indicated by the pointer. In the other condition (NID) the pointer appeared with the display to control for field complexity but was irrelevant for detecting the target form. The pointer appeared an equal number of times at all display locations for each location of the target form in the NID condition. Ss always knew the relevance of the indicator prior to experimental sessions.

Four experimental conditions were determined by combining the two indicator conditions with the two exposure durations. These conditions were systematically randomized among Ss so that each condition appeared an equal number of times at each stage of practice. Under each of these four conditions, an $\mathrm{S}$ made 64 judgments to each of the six display sizes. The randomization of the order of stimulus presentations was further constrained within sets of 384 judgments so that the target form appeared an equal number of times to the right and left. All Ss received the same random order. Each $S$ was tested for 16 experimental sessions, 4 sessions for each of the four conditions.

\section{RESULTS}

The number of correct detections was entered into a five-way repeated-measures analysis of variance (exposure durations, indicator relevance, display size, location of target form, and Ss).

As would be expected from the pretraining procedures, $\mathrm{Ss}$ ' detection judgments were more accurate at the $90 \%$ exposure duration than at the $75 \%(\mathrm{~F}=155.4, \mathrm{df}=1 / 7, \mathrm{p}<.001)$. Detection accuracy was greater in the ID condition than in the NID $(\mathrm{F}=24.2, \mathrm{df}=1 / 7, \mathrm{p}<.001)$.

Figure 1 shows the obtained relations among exposure duration, indicator condition, and display size. The significant interaction of indicator relevance and display size $(\mathrm{F}=18.0$, $\mathrm{df}=5 / 35, \mathrm{p}<.001)$ is shown clearly in the figure by the increasing difference between ID and NID slopes for both SF HR conditions as display size increases.

The interaction of target location and size $(F=1.69$, $\mathrm{df}=35 / 245, \mathbf{p}<.01)$ is illustrated in Fig. 2 . Forms are detected most accurately when in a right or left relationship to the fixation point, the 67.5-, 112.5-, 247.5-, and 292.5-deg locations. Differences increase with increases in the number of stimulus forms.

The distribution of errors made at the different locations as a function of indicator position was examined to determine whether Ss were influenced significantly by the indicator in the NID condition. An examination of the errors revealed no tendency for errors to vary as a function of the indicator in any systematic fashion.

Estimates of the average number of forms processed from NID displays were made on the basis of the Estes and Taylor (1966) serial scanning model assumptions. In the framework of the Estes and Taylor model, the probability of a correct response, $\mathrm{p}_{\mathrm{C}}$, is predicted from the following equation:

$$
\mathrm{P}_{\mathrm{C}}=\frac{\mathrm{P}}{\mathrm{D}}+\left(1 \quad \frac{\mathrm{P}}{\mathrm{D}}\right)^{1 / 2} \text {, }
$$

where $P$ represents the number of forms effectively processed, or noted, and $D$ the number of stimulus forms.

Since the target forms are randomly placed in the displays, the probability that the target form falls among the $\mathbf{P}$ forms perceived is $P / D$, and whenever the target form is not perceived, the probability of a correct response is $1 / 2$. Replacing $\mathrm{p}_{\mathrm{C}}$ by the observed proportion of correct detections at a given display size, an estimate of $\mathbf{P}$ in terms of empirical results is provided by the equation:

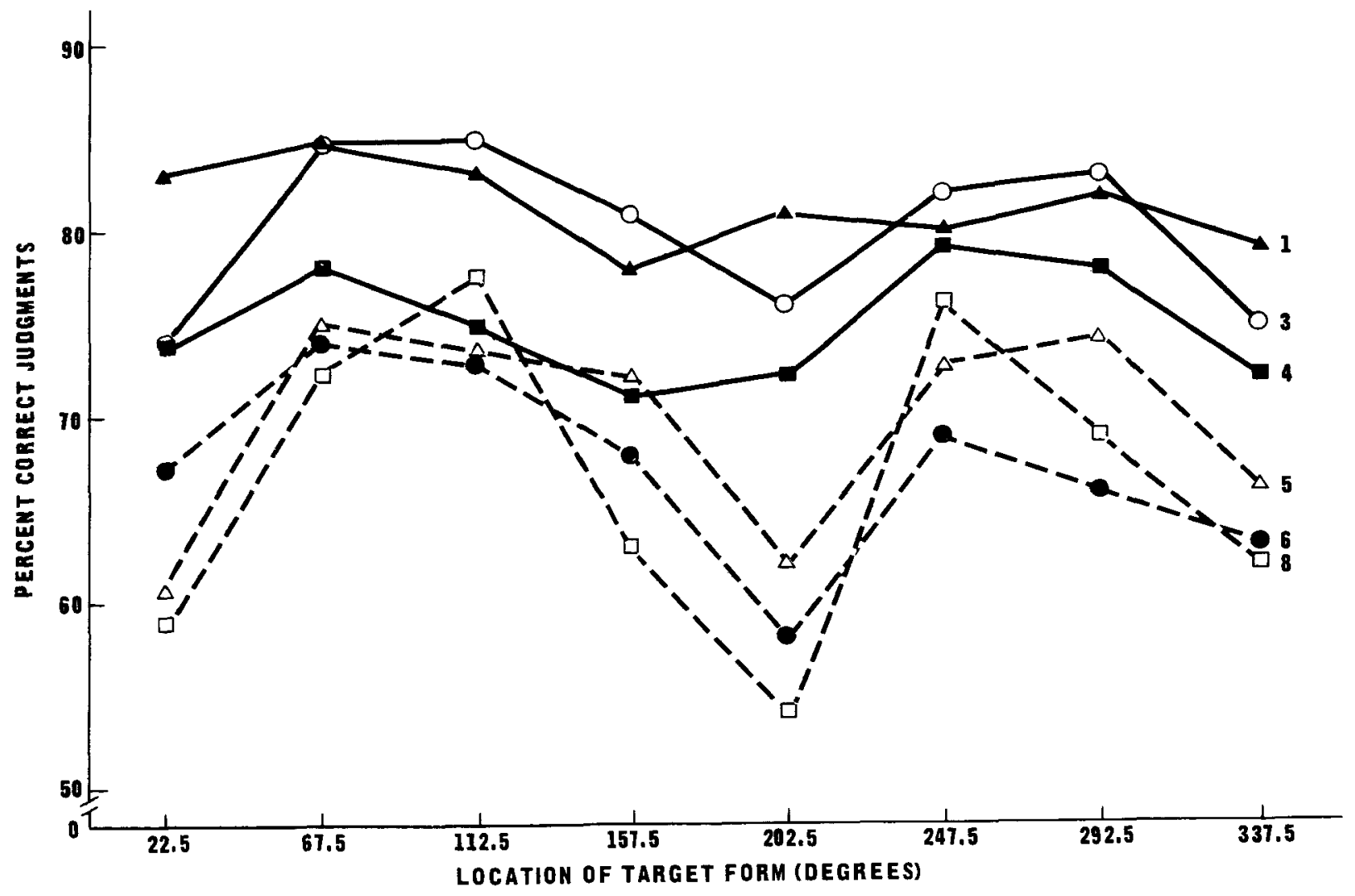

Fig. 2. Per cent correct judgments as a joint function of the number of display forms and target-form location. 
Table 1

Estimates of the Mean Number of Forms ( $\hat{\mathbf{P}})$ and Mean Number of Sensory Channels $(\hat{\mathbf{K}})$ Noted

\begin{tabular}{|c|c|c|c|c|c|c|}
\hline \multirow[t]{2}{*}{$\begin{array}{l}\text { Number of } \\
\text { Forms }\end{array}$} & \multirow[b]{2}{*}{$\begin{array}{c}\text { NID } \\
\text { HR }\end{array}$} & \multicolumn{2}{|c|}{ 75\% HR Duration } & \multicolumn{3}{|c|}{ 90\% HR Duration } \\
\hline & & $\hat{\mathbf{P}}$ & $\hat{\mathbf{K}}$ & $\underset{\text { HR }}{\text { NID }}$ & $\hat{\mathbf{P}}$ & $\hat{\mathbf{K}}$ \\
\hline 1 & .767 & .534 & 1.000 & .892 & .784 & 1.000 \\
\hline 3 & .734 & 1.404 & 2.6 & .840 & 2.040 & 2.602 \\
\hline 4 & .636 & 1.248 & 2.337 & .799 & 2.392 & 3.051 \\
\hline 5 & .615 & 1.150 & 2.154 & .695 & 1.950 & 2.487 \\
\hline 6 & .586 & 1.032 & 1.932 & .666 & 1.992 & 2.541 \\
\hline 8 & .564 & 1.024 & 1.918 & .608 & 1.728 & 2.204 \\
\hline
\end{tabular}

$$
\hat{\mathbf{P}}=\left(2 \mathrm{p}_{\mathrm{C}}-1\right) \mathrm{D} .
$$

Using this procedure, the obtained proportions of correct detections pooled over the eight $\mathrm{Ss}$ have been substituted for $\mathrm{PC}$ to provide estimates of the mean number of forms processed per display for each display size. These values are shown in Table 1. Estimates of $\hat{\mathbf{P}}$ first increase and then decrease as a function of display size.

The scanning model expectation of a systematic increase in $\mathbf{P}$ as a function of increased display size is not found, and estimates are well below those found by Estes and collaborators, and Sperling (1960).

If it is assumed that the SF HR corrected for chance estimates the availability of the form to the noting mechanism, low estimates are to be expected. The average number of forms available on a given trial should be equal to $\mathrm{Np}_{A}$ where $\mathrm{N}$ equals the number of forms, and $\mathrm{p}_{\mathrm{A}}$ equals the probability of a form being available, or $\mathrm{HR}-(1-\mathrm{HR})$. The maximum average number of forms available for the $75 \%$ and $90 \%$ durations would then be four and six, respectively, for the eight-form displays. The table shows that $P$, which can be viewed as an estimate of number of available forms noted, reaches a maximum of 1.4 and 2.4 for the $75 \%$ and $90 \%$ durations, respectively.

Estimates of the number of channels within a parallel scan can also be made. If it is assumed that each channel has associated with it uncorrelated internal perceptual system noise, the SF HR corrected for chance represents the probability of any form being correctly available to the noting mechanism on a given trial. The formula for predicting per cent correct detections is as follows:

$$
\mathrm{p}_{\mathrm{C}}=\frac{\mathrm{K}}{\mathrm{D}} \mathrm{p}_{\mathrm{A}}+\frac{\mathrm{K}}{\mathrm{D}}\left(1-\mathrm{p}_{\mathrm{A}}\right)^{1 / 2}+\frac{\mathrm{D}-\mathrm{K}}{\mathrm{D}}(1 / 2)
$$

where $\mathrm{K}$ is equal to the number of parallel channels, $\mathrm{p}_{\mathrm{A}}$ represents the probability of a form being available, and $D$ equals number of display forms. The model assumes $S$ guesses correctly as frequently as incorrectly on those trials on which the critical information is not available. The maximum value of $K$ is $D$. When $K$ equals $D, p_{C}$ is equal to the SF HR. Substituting the observed proportion of correct response as an estimate of $\mathrm{pC}_{\mathrm{C}}$ and solving the above equation for $\mathrm{K}$, the formula becomes:

$$
\hat{\mathrm{K}}=\frac{\left(2 \mathrm{p}_{\mathrm{C}}-1\right) \mathrm{D}}{\mathrm{p}_{\mathrm{A}}}
$$

The estimated values are presented in the second column of Table 1. This formula predicts identical slopes to Formula 1. Only the $y$ intercepts differ. The parallel processing model predicts equivalent values of $K$, but not of $P$, regardless of exposure duration, since rate of processing is not a limiting factor under the parallel processing hypothesis.

\section{DISCUSSION}

The principal finding in this study was that when foveal sensitivity was controlled and response uncertainty remained constant, the ability of an $S$ to detect a brief visual form varied with attentional demands. Of particular significance was the nature of the difference in processing when attentional demands differed. Detection was more accurate when an indicator designated the target form than when the indicator was irrelevant, and the differences increased with increasing display size.

These findings support previous evidence of the operation of a selective attentional process in visual perception (Eriksen \& Lappin, 1967), and also indicate some limitations to such a process. Even when the target form was indicated, decreases in HR were observed with the presence of additional forms.

If items of information are read out one by one from a wholly intact brief buffer storage until the sensory trace decays (e.g., Sperling, 1960; Aaronsen, 1967), and processing is limited only by rate of noting, the advantage of an indicator would be to select the target form as the first form to be noted. If noting speed were the only processing limitation, we could not account for the ID-condition HR decrease.

In the NID condition, where the indicator is irrelevant and noting of nontarget forms is necessary for efficient performance, we find evidence of display interference apart from noting requirements. The addition of forms produces decreases in estimates of $\mathbf{P}$ and $\mathbf{K}$ as the number of forms surpasses three in the $75 \%$ SF HR condition and four in the $90 \%$.

The ID and NID results combined indicate display in terference effects in visual detection. One possibility is that the addition of new forms reduces the detectability of any single form by means of some disturbance of the attentional field. Neisser (1967) discusses the possibility of a preattentional mechanism that segregates forms prior to noting. Cluttering of the field may disrupt such a mechanism.

Another possibility is that an interaction occurs at the sense organ level. However, previous evidence suggests that this interaction is unlikely with 1 -deg separation between forms (Collins \& Eriksen, 1968).

Whatever this interference, differentiation between serial and parallel models of the noting mechanism with the present methodology is impossible unless the reduction in accuracy is taken into consideration. In an attempt to take display interference error into account, the assumption was made that ID-condition $H R$ reflects the identifiability of a form in a sensory channel that has been selected for analysis by the noting mechanism. This HR then includes both perceptual system error and display interference error. The divergence of ID and NID slopes then estimates the HR reduction due to increasing noting requirements. In this framework, it is possible to treat the NID $H R$ as reflecting three primary limitations:

(1) Internal perceptual system noise.

(2) Display interference.

(3) Noting capacity, which may be limited either by rate of noting, or by the number of parallel sensory channels.

To estimate the average number of sensory channels from which information, correctly or incorrectly, is noted, we can replace the $p_{A}$ in Eq. 4 above by the $p_{A}$ represented by the ID-condition HR for each display size, which takes into account both perceptual system noise and display interference. In this equation $p_{A}$ thus represents the probability of a form within the perceptual scan being correctly noted. Estimates of $\mathrm{K}$ based on this formula are given in Table 2 .

Even when display interference error is roughly considered, limits to the perceptual span are found, as indicated by Table 2-limits well under previous estimates for the display sizes used in this investigation. The most notable change produced by 
Table 2

Estimates of Mean Number of Sensory Channels Noted When $P_{A}$ is Estimated by ID HR

\begin{tabular}{lcccc}
\hline $\begin{array}{c}\text { Number of } \\
\text { Forms }\end{array}$ & $\mathbf{P}_{\mathbf{A}}$ & $\begin{array}{c}\text { 75\% HR Duration } \\
\mathbf{K}\end{array}$ & $\begin{array}{c}\text { 90\% HR Duration } \\
\mathbf{P}_{\mathbf{A}}\end{array}$ \\
\hline 1 & .460 & 1.161 & .742 & $\mathbf{K}$ \\
3 & .532 & 2.639 & .722 & 1.057 \\
4 & .386 & 3.233 & .710 & 2.825 \\
5 & .368 & 3.125 & .578 & 3.369 \\
6 & .360 & 2.867 & .536 & 3.373 \\
8 & .394 & 2.599 & .602 & 3.716 \\
\hline
\end{tabular}

the above procedure is the increased similarity between $75 \%$ - and $90 \%$-exposure estimates for the same display size, except for the six-form displays.

Interestingly, in the Estes and Taylor (1966) investigation, all analyses involving only a single display size yielded results that could be nicely interpreted both qualitatively and quantitatively in terms of a fixed-sample-size model (Estes \& Taylor, 1964). On the other hand, the observed function relating mean number of forms perceived to display size was quite unaccountable in terms of this model. Table 2 shows results that are interpretable in terms of a fixed sensory-channel-size model for a given display size, in that estimates of $\mathrm{K}$ are very similar for both the $75 \%$ and $90 \%$ durations. However, values of $\hat{\mathrm{K}}$ increase with increased display size, and then decrease.

These somewhat paradoxical findings could be reconciled by interpretation in terms of an attentional mechanism that varies in the number of sensory channels in its scan, as a function of the spatial distribution of elements. The findings of significant spatial selectivity effects supports this view. Selection of forms for larger display sizes was determined to a substantial extent by the location of the forms in the display. It is possible that spatial selectivity factors may be reflecting some selective preattentional process. If such a process does occur, the results of Table 2 are interpretable in terms of either serial or parallel processing mechanisms. It is apparent that display distribution and noting requirement influences need further investigation in order to determine adequately their role in the scanning process.

The values given in Tables 1 and 2 are based on a chain of inferences with in the framework of a particular model and the obtained experimental results, and several models could account for the observed pattern of results. It is, therefore, difficult to arrive at any general conclusions concerning the nature of the noting mechanism. It is significant that previous studies supporting both serial and parallel processing mechanisms have not found perceptual span limitations similar to those found in the NID conditions. The one methodological difference that separates this study both from the work of Estes and collaborators and Eriksen and collaborators is the use of single-featured stimuli, rather than multifeatured letters. A recent investigation employing single-featured stimuli (Keeley \& Doherty, 1968) has supported the notion that if parallel processing occurs, it occurs at the level of features. If this is true, it would account for the large difference between the results of this investigation and those obtained by Eriksen (1966) and Collins \& Eriksen (1968), which were interpreted as supporting the simultaneous processing of up to four forms. It would seem that estimates of perceptual span, and models of serial vs parallel processing based on such estimates, which do not consider the dimensionality of the forms, as well as possible influences due to display distribution, are highly suspect.

\section{REFERENCES}

AARONSON, D. Temporal factors in perception and short-term memory. Psychological Bulletin, 1967,67, 130-144.

COLLINS, J. F., \& ERIKSEN, C. W. The perception of multiple simultaneously presented forms as a function of foveal spacing. Perception \& Psychophysics, 1967, 2, 369-373.

ERIKSEN, C. W. Independence of successive inputs and uncorrelated error in visual form perception. Journal of Experimental Psychology, 1966 72, 26-35.

ERIKSEN, C. W., \& LAPPIN, J. S. Selective attention and very short-term recognition memory for nonsense forms. Journal of Experimental Psychology, 1967, 73, 358-364.

ESTES, W. K., \& TAYLOR, H. A. A detection method and probabilistic models for assessing information processing from brief visual displays. Proceedings of the National Academy of Science, 1964,52 , No. 2 , 446-454.

ESTES, W. K., \& TAYLOR, H. A. Visual detection in relation to display size and redundancy of critical elements. Perception \& Psychophysics, $1966,1,9-16$.

ESTES, W. K., \& WESSELL, D. L. Reaction time in relation to display sizc and correctness of response in forced-choice visual signal detection. Perception \& Psychophysics, 1966, 1, 369-373.

KEELEY, S. M., \& DOHERTY, M. E. Simultaneous and successive presentations of single-featured and multiple-featured forms: Implications for the parallel processing hypothesis. Perception \& Psychophysics, $1968,4,296-298$.

NEISSER, U. Cognitive Psychology. New York: Appleton-Century, 1967. SPERLING, G. The information available in brief visual presentations. Psychological Monographs: General \& Applied, 1960, 74, No. 11 (Whole).

SPERLING, G. A model for visual memory tasks. Human l'actors, 1963.5, 19-31.

\section{NOTES}

1. This paper was based on a dissertation submitted to the Psychology Department of the University of Illinois in partial fulfillment of the requirement for the $\mathrm{PhD}$ degree. The writer wishes to thank Charles $\mathrm{W}$. Eriksen for his helpful supervision. This research was supported in part by Grant MH-01 206 of the United States Public Health Service.

2. Address: Bowling Green State University, Bowling Green, Ohio 43402.

(Accepted for publication January 13, 1969.) 